\title{
EDITORIAL
}

\section{LOOKING TO THE FUTURE}

Microbiology, as a research field, is undergoing unprecedented growth. The challenge now is to channel this effort and commitment into addressing the big questions facing the discipline.

It is one year since the inaugural issue of Nature Reviews Microbiology landed on the desks of microbiologists around the world - a relatively long time in the ongoing development of, arguably, the fastest-moving discipline in the life sciences. In the time frame of 12 issues of Nature Reviews Microbiology, some 75,000 articles were published by scientists striving to understand the biology of microbial life. It is standard in such 'birthday' discourses to reflect on developments in the field since issue one of the journal - analyse the trends, select the highlights, and congratulate the movers and shakers of the past 12 months. A more interesting exercise, however, is to ponder the following: what if we were to carry out such a task on our 10th anniversary - what highlights would we expect to be celebrating in the 121st issue of Nature Reviews Microbiology?

In an effort to answer this question, Nature Reviews Microbiology conducted an informal poll of its readers to assess what they thought were the challenges facing microbiologists over the next decade and, although this survey was far from representative, there was considerable consensus over the major challenges facing our community. First, and not surprisingly, it was felt that there is a compelling need for a greater understanding of both the diversity of microorganisms and how this diversity evolved. More than $99 \%$ of microbial species remain uncultured, and bacterial and archaeal phylogeny is complicated by horizontal genetic exchange. And, although the immense diversification of microbial lineages is well established, one basic question that is easily asked but very challenging to address is what were the specific events that led to these diversifications? At a more practical level, a better understanding of the diversity of pathogens is required, including knowledge of the natural reservoirs of virulence determinants and mechanisms for their acquisition in the environment.

According to our survey, another area of study that requires attention is commensalism. In the example of the mammalian gut, we have much to learn about the diversity of our endogenous microbial community and the degree of variability within this diversity. It is also imperative that we gain a fuller appreciation of the relationship between the diversity of our microflora and human health and disease. On the challenges facing microbiologists and clinicians dealing with infectious diseases, an unmet need highlighted by the poll is the issue of detection and diagnosis of microbial infection. One obvious consequence of progress in this area is the inappropriate use of therapeutics and the consequent development of antibiotic resistance. Additional challenges for the next decade in the field of medical microbiology include a greater understanding of host susceptibility, postulating and proving microbial aetiologies for chronic disease and identifying novel correlates of protective immunity. Moving from human health to our planet's health, focusing research efforts on the interplay between the biosphere, dominated by microorganisms, and the geosphere promises enormous dividends in understanding the history of the Earth and accurately predicting its future.

So, the challenges are there - but is the technology available to address these challenges? The much-vaunted power of functional genomics has yet to be fully realized but this approach has already led to major advances and, undoubtedly, will continue to do so. One other technological approach that will prove hugely beneficial is the ability to analyse the biology of single cells in the context of their populations. Of course, the various challenges that face microbiologists over the coming years require more than just technological advances. Addressing these questions will require interdisciplinary interaction and computational expertise - attributes that need to be factored into the training of the next generation of microbiologists. As always, adequate funding will also be required and this in turn depends on giving the required attention to public education to counteract the 'who cares?' attitude of many of our public representatives. The rewards for these efforts, however, will be substantial and could potentially be realized relatively quickly. Indeed, browsing through the table of contents of the 121st issue of Nature Reviews Microbiology could make very interesting reading. 